\title{
Utilidad del fenotipo hipertensión-obesidad abdominal para identificar personas con síndrome metabólico
}

\section{Usefulness of the hypertension-abdominal obesity phenotype to identify people with metabolic syndrome}

\author{
Eduardo Cabrera-Rode ${ }^{1 *}$, Ana C. Borja-Coronel ${ }^{2}$, Rocío Montes de Oca-Somoano², Janet Rodríguez-Acosta ${ }^{1}$, \\ Ileana Cubas-Dueñas ${ }^{1}$, Yuri Arnold-Domínguez ${ }^{3}$, José Hernández-Rodríguez ${ }^{3}$ y Oscar Díaz-Díaz ${ }^{3}$
}

${ }^{1}$ Departamento de Inmunología; ${ }^{2}$ Servicio de Endocrinología; ${ }^{3}$ Centro de Atención a la Diabetes. Instituto de Endocrinología, La Habana, Cuba

\section{RESUMEN}

El objetivo del presente estudio fue determinar si el fenotipo hipertensión-obesidad abdominal (FHTOA) es el binomio más útil para identificar personas con síndrome metabólico (SM). Estudio observacional, descriptivo y de corte transversal que incluyó 350 personas con edades comprendidas entre 19 y 70 años, reclutadas consecutivamente en una consulta habilitada para esta investigación de sujetos con exceso de peso. Como criterio diagnóstico de personas con SM se empleó la definición de la declaración provisional conjunta, además se estudiaron los fenotipos FHTOA, hipertrigliceridemia-obesidad abdominal (FHGOA) e hiperglucemia-obesidad abdominal (FHGLOA). La concordancia entre los criterios de SM y los fenotipos estudiados se estimó con el coeficiente kappa; también se calculó la sensibilidad y especificidad de los fenotipos para identificar personas con SM. La frecuencia del FHTOA fue del $76.9 \%$ (269/350) y la frecuencia del SM fue del $69.1 \%$ (242/350), le siguen el FHGOA (28.0\%) y el FHGLOA (20.9\%). El FHTOA mostró alta sensibilidad (90.5\%) y moderada concordancia (0.475) para detectar personas con SM. La alta sensibilidad y la moderada concordancia del FHTOA para identificar a las personas con SM, así como la sencillez en su determinación, lo hacen una opción útil para detectar personas con SM.

Palabras clave: Obesidad. Sobrepeso. Síndrome metabólico. Hipertensión. Obesidad abdominal.

\begin{abstract}
The objective of the present study was to determine if the hypertension-abdominal obesity phenotype (HTAOP) is the most useful binomial to identify people with metabolic syndrome (MS). Observational, descriptive and cross-sectional study that included 350 subjects with excess weight between 19 and 70 years old, who were consecutively recruited in a consultation enabled for this investigation. The definition of the joint provisional declaration was used as a diagnostic criterion for MS. In addition, the HTAOP, the hypertriglyceridemia-abdominal obesity phenotype (HGAOP) and the hyperglycaemia-abdominal obesity phenotype (HGLAOP) were also studied. The concordance between the MS criteria and the phenotypes studied was estimated with the kappa coefficient; the sensitivity and specificity of phenotypes to identify people with MS were also calculated. The frequency of HTAOP was $76.9 \%$ (269/350) and the frequency of MS was $69.1 \%$ (242/350), followed by the HGAOP (28.0\%) and the HGLAOP (20.9\%). The HTAOP showed high sensitivity (90.5\%) and moderate concordance (0.475) to detect people with MS. The high sensitivity and moderate concordance of the HTAOP to identify people with MS, as well as the simplicity in its determination, make it a useful option for detecting people with MS.
\end{abstract}

Key words: Obesity. Overweight. Metabolic syndrome. Hypertension. Waist circumference.
Correspondencia:

*Eduardo Cabrera-Rode

E-mail: eduardo.cabrerarodedgmail.com
Fecha de recepción: 31-12-2019

Fecha de aceptación: 03-04-2020

DOI: 10.24875/ALAD.20000413
Disponible en internet: 18-05-2020 Rev ALAD. 2020;10:131-42

2248-6518 / @ 2020 Asociación Latinoamericana de Diabetes. Publicado por Permanyer. Este es un artículo open access bajo la licencia CC BY-NC-ND (http://creativecommons.org/licenses/by-nc-nd/4.0/). 


\section{INTRODUCCIÓN}

El síndrome metabólico (SM) es una entidad en la que se asocian varios factores (intolerancia a la glucosa, alteraciones lipídicas, hipertensión arterial [HTA] y obesidad abdominal) precursores de enfermedad cardiovascular (ECV) y de diabetes mellitus tipo $2(\mathrm{DM} 2)^{1-3}$.

No existen datos estadísticos globales robustos sobre la prevalencia del SM, debido a que es muy difícil conocer su frecuencia real mundial, pues se utilizan al menos seis definiciones diferentes ${ }^{1,4}$. El SM es una afección muy común, que afecta del 20 al 35\% de la población mundial adulta (frecuencia estimada cuando se utiliza alguna de las seis definiciones en población adulta)1,4-6.

El SM se presenta con amplias variaciones fenotípicas, debidas a las diversas combinaciones de sus componentes: HTA, obesidad abdominal, hipertrigliceridemia, hiperglucemia y colesterol de lipoproteínas de alta densidad (C-HDL) bajo, en personas con una predisposición genética determinada, condicionada, a su vez, por factores ambientales ${ }^{1,6,7}$.

En el 2010, la consulta de expertos de la Organización Mundial de la Salud (OMS) declaró que el SM podría considerarse útil como un concepto educativo y que tiene una utilidad práctica limitada como herramienta de diagnóstico o de gestión. El SM es una afección de riesgo cardiometabólico más que un diagnóstico clínico, por lo que debería excluir a los individuos con diabetes establecida o ECV conocida ${ }^{8}$.

El riesgo de ECV asociado al SM aparentemente no es superior al de cada uno de sus componentes y el valor para predecir diabetes del SM no es superior al de la glucosa en ayunas 9 . Por ende, varios investigadores9,10 han declarado que se debe evitar etiquetar a las personas con el término de SM, que los adultos con cualquier componente del SM deben ser evaluados para la existencia de otro factor de riesgo de ECV o de diabetes y que estos deben ser tratados de forma individual y agresiva. Por lo antes expresado, se hace ineludible investigar varias combinaciones de binomios de los componentes del SM que sean eficientes para identificar personas con el llamado SM.

Las variaciones fenotípicas clínicas pueden analizarse de forma simple o combinada, según los componentes del SM. De todos los componentes del síndrome, la obesidad es el más importante, debido a su relación con la aparición de alteraciones del metabolismo de la glucosa y la dislipidemia ${ }^{1,6,7,11,12}$. Además, representa un factor de riesgo independiente de ECV, el cual se acentúa cuando la adiposidad es predominantemente central.

En la actualidad, se utiliza la circunferencia de la cintura (CC) para estimar la grasa abdominal; la CC se considera como un componente fenotípico importante del $S M^{1,3,7,11}$. Según De Oliveira ${ }^{13}$, la $C C$ y el índice de masa corporal (IMC) se asocian mejor con la HTA, que los índices de adiposidad corporal y de adiposidad visceral (indicador de la función del tejido adiposo visceral), por lo que la CC y el IMC podrían ser indicadores útiles para detectar personas con riesgo de $\mathrm{HTA}^{13}$.

Las mediciones antropométricas son fáciles de realizar, efectivas y económicas; se emplean en la pesquisa inicial de obesidad, HTA y de otras alteraciones metabólicas ${ }^{13-15}$. El IMC se utiliza para identificar el sobrepeso y la obesidad, mientras que el índice cintura-cadera y la CC se emplean como indicadores de adiposidad corporal central ${ }^{14,15}$.

Se han investigado distintos métodos para realizar la pesquisa del SM y simplificar la práctica clínica. EI 
fenotipo hipertrigliceridemia-obesidad abdominal (FHGOA) ha sido el binomio más estudiado de los componentes del SM y se ha reafirmado como predictor de riesgo cardiovascular, mediante su asociación con alteraciones metabólicas, entre las que figuran: la alteración del perfil de lípidos, la HTA, la DM2 y la resistencia a la insulina. El FHGOA también se ha reconocido como una herramienta que apoya la detección sencilla y menos costosa de estas enfermedades ${ }^{16-21}$.

Para simplificar aún más la pesquisa del SM, se ha propuesto realizar en esencia la medición de la CC y la tensión arterial (TA), dos parámetros que se evalúan más fácilmente en la práctica diaria que las mediciones bioquímicas ${ }^{21}$. En Rumania, en población general, se investigaron varios métodos para realizar la pesquisa del SM para simplificar la práctica clínica; los autores han estudiado la combinación de varios binomios de los componentes del $\mathrm{SM}^{21}$. Los mismos autores han encontrado el fenotipo hipertensión-obesidad abdominal (FHTOA) como la combinación más frecuente en personas con $\mathrm{SM}^{21}$. Nita, et al. ${ }^{21}$ mostraron que las personas con el FHTOA tuvieron 6.7 veces mayor probabilidad de presentar SM en comparación con las personas sin FHTOA.

En la literatura internacional revisada existe un solo estudio en población general que empleó el FHTOA para identificar personas con $\mathrm{SM}^{21}$. Al mismo tiempo, no hemos encontrado ninguna investigación que use el FHTOA en sujetos con sobrepeso y obesos para evaluar la efectividad de este binomio en la identificación de personas con SM, lo que sugiere que esta relación no se ha empleado frecuentemente en la práctica médica.

En Cuba, la alta prevalencia de los principales factores de riesgo cardiovascular, tales como la HTA (30.9\%), el sobrepeso (44.8\%), la obesidad (15.0\%), las alteraciones lipídicas (24.1\%) y la diabetes mellitus (10.0\%) $)^{22-24}$, repercuten en el incremento de la mortalidad por enfermedades cardiovasculares (2016-2017) $)^{25,26}$, con tasas de 145-5-156.7 por 100,000 habitantes respectivamente ${ }^{22}$. En un área de salud de Cienfuegos (Cuba) un estudio encontró que la HTA también fue el componente más prevalente del $\mathrm{SM}^{27}$. Este síndrome se ha incrementado en las últimas décadas, tanto en Cuba (21.7-27.2\%) $)^{27,28}$ como en América Latina (13.7-27.0\%) ${ }^{29}$ y América Central $(23.0-35.1 \%)^{30}$.

Puesto que en Cuba se ha observado un incremento de los factores de riesgo cardiovascular, y que la prevalencia del SM se eleva cuando se incrementa el IMC ${ }^{1,5,7,31}$, propusimos determinar si el FHTOA es el binomio más útil de los conocidos para identificar personas con SM en adultos con sobrepeso y obesos.

\section{MATERIAL Y MÉTODOS}

Se realizó un estudio observacional, descriptivo y de corte transversal. Se estudiaron 350 sujetos no diabéticos con sobrepeso (IMC entre 25,0 y $29,9 \mathrm{~kg}$ / $\mathrm{m}^{2}$ ) y obesos $\left(I M C \geq 30,0 \mathrm{~kg} / \mathrm{m}^{2}\right)$ según los criterios de la $\mathrm{OMS}^{32}$, con edades comprendidas entre los 19 y 70 años, que fueron reclutados consecutivamente de una consulta de investigación habilitada para este estudio en el periodo comprendido entre los años 2014 y 2015, en la Habana, Cuba. Se excluyeron las personas con diabetes tipo 1 y 2, así como con otros tipos específicos de diabetes y a embarazadas, además de individuos con enfermedades crónicas asociadas que requirieron tratamiento esteroideo o inmunosupresor.

La selección de la muestra se realizó entre personas que aceptaron participar en el estudio realizado en campañas de salud de despistaje de factores de riesgo metabólico en la Habana. Se calculó el 
tamaño muestral mediante el programa Epidat 3.1. Con una población de 4,500, un nivel de confianza al 95\%, una proporción esperada de obesos del $31.6 \%$, una precisión de muestreo de 0.9 y un efecto de diseño de 0.1; se estimó un mínimo de 313 sujetos que incluir en el estudio.

Para el diagnóstico de SM se empleó como criterio la definición de la declaración provisional conjunta (JIS, Joint Interim Statement) de varias asociaciones (Federación Internacional de Diabetes [IDF], American Heart Association, Federación Mundial del Corazón, Sociedad Internacional de Ateroesclerosis, Asociación Internacional para el Estudio de la Obesidad y el National Heart Lung and Blood Institute) ${ }^{3,33}$. Se definió como la presencia de al menos tres de los siguientes componentes: CC en hombres $\geq 90 \mathrm{~cm}$ y en mujeres $\geq 80 \mathrm{~cm}^{3,33,34}$; triglicéridos $\geq 1.7 \mathrm{mmol} / \mathrm{l}$ (150 mg/dl) o tratamiento para la dislipidemia, C-HDL en hombres $<1.03 \mathrm{mmol} / \mathrm{l}$ (40 $\mathrm{mg} / \mathrm{dl}$ ) y en mujeres $<1.29 \mathrm{mmol} / \mathrm{l}$ (50 mg/dl) o tratamiento para la dislipidemia3,33, presión arterial $\geq 130 / 80 \mathrm{mmHg}$ o tratamiento antihipertensivo ${ }^{35}$, y glucosa en ayunas $\geq 5.6$ $(100 \mathrm{mg} / \mathrm{dl})$ o tratamiento para la hiperglucemia3,33.

El FHTOA se definió como la presencia de presión sistólica $\geq 130 \mathrm{mmHg}$ o presión diastólica $\geq 80 \mathrm{mmHg}$ o tratamiento antihipertensión ${ }^{35}$, más una CC $\geq 80 \mathrm{~cm}$ en mujeres $y \geq 90 \mathrm{~cm}$ en hombres 3333,34 .

El FHGOA se definió como la presencia de triglicéridos $\geq 1.7 \mathrm{mmol} / \mathrm{l}(150 \mathrm{mg} / \mathrm{dl})$ o tratamiento para la dislipidemia ${ }^{17,21}$, más una $C C \geq 80 \mathrm{~cm}$ en mujeres $\mathrm{y}$ $\geq 90 \mathrm{~cm}$ en hombres 333,34 .

El fenotipo hiperglucemia-obesidad abdominal (FHGLOA) ${ }^{21}$ se definió como la presencia de glucosa en ayunas $\geq 5.6 \mathrm{mmol} / \mathrm{l}(100 \mathrm{mg} / \mathrm{dl}) \mathrm{y} \leq 6.9 \mathrm{mmol} / \mathrm{l}$ (125 mg/dl) o tratamiento para la hiperglucemia, más una $\mathrm{CC} \geq 80 \mathrm{~cm}$ en mujeres $\mathrm{y} \geq 90 \mathrm{~cm}$ en hombres $3,33,34$.
A todos los sujetos se les aplicó un cuestionario para la recolección de datos que incluyó edad, sexo, medicamentos empleados, variables antropométricas (peso, talla, IMC y CC) y clínicas (TA) en el formato diseñado para el estudio, además de los antecedentes personales de HTA. El médico investigador se encargó de facilitar las respectivas historias para la recolección de los valores bioquímicos.

En todos los sujetos con sobrepeso y obesos se determinó la presencia de SM según los criterios $\mathrm{JIS}^{3}$ y la de los binomios de los componentes del SM anteriormente descritos. La definición de la JIS fue considerada como el estándar de oro para el diagnóstico del SM.

Los sujetos fueron evaluados según peso, talla, CC e IMC (peso [kg]/talla $\left[\mathrm{m}^{2}\right]$ ). La medida de la CC se tomó con una cinta métrica con el sujeto colocado de pie, en espiración, con el abdomen relajado, tomando como referencia el punto medio entre el borde inferior de la última costilla y la espina ilíaca anterosuperior de cada lado. En los casos de abdómenes péndulos la medición se realizó en el punto más prominente del abdomen.

La medición de la TA a cada sujeto se realizó con esfigmomanómetro con mango de acorde al tamaño del brazo. Previamente, el sujeto estuvo sentado en reposo durante 10 minutos. El procedimiento se realizó tres veces en el brazo derecho, con un intervalo de cinco minutos. El valor final de la TA correspondió al promedio de las tres mediciones obtenidas.

Las determinaciones bioquímicas se realizaron a cada sujeto en el momento de la obtención de la primera muestra sanguínea (basal), luego de aproximadamente 8-12 horas de ayuno. Se midieron en ayunas las concentraciones plasmáticas de glucosa y lípidos (triglicéridos y C-HDL). 
La concentración de glucosa en ayunas, triglicéridos y C-HDL se midieron en los sujetos mediante un analizador automático (Elimat, Francia) por métodos enzimáticos.

\section{ANÁLISIS ESTADÍSTICOS}

Para los cálculos estadísticos se utilizó el programa SPSS versión 21 para Windows. Se realizó un análisis descriptivo de la muestra expresando las variables en sus respectivas medidas de resumen: las cualitativas en cifras relativas y absolutas (frecuencias y porcentajes), y las cuantitativas en sus medidas de posición y dispersión (media y desviación estándar). Para determinar la asociación entre las variables cualitativas se usó la prueba de Chi cuadrada de Pearson.

El grado de concordancia entre los criterios diagnósticos de SM según JIS y de los tres fenotipos estudiados (FHTOA, FHGOA y FHGLOA) fue calculado por el coeficiente kappa de Cohen. La interpretación del coeficiente kappa se realizó con base en los seis niveles de fuerza de concordancia propuestos por Landis y Koch ${ }^{36}: \leq 0.00$ (pobre); 0.01-0.20 (leve); 0.21-0.40 (aceptable), 0.41-0.60 (moderada); 0.61-0.80 (considerable) y 0.81-1.00 (casi perfecta). Se realizó también el cálculo de la sensibilidad, especificidad, valores predictivos positivos (VPP) y negativos (VPN) para evaluar la capacidad de los fenotipos (FHTOA, FHGOA y FHGLOA) de identificar la presencia de SM.

En todos los casos se trabajó para un nivel de confianza del 95\% (IC 95\%), prefijando un error alfa de 0.05 y una región crítica o de rechazo a $p=0.05$, de tal forma que cuando $p<0.05$ existió significación estadística.

El proyecto fue aprobado por el Comité de Ética e Investigación del Instituto de Endocrinología,
Universidad de Ciencias Médicas de La Habana. Los participantes del estudio dieron su consentimiento informado por escrito, previa información de los objetivos y propósitos del estudio. Todos los datos se mantuvieron confidenciales y la identidad del participante se mantuvo en el anonimato.

\section{RESULTADOS}

Se estudiaron 350 sujetos con exceso de peso entre 19 y 70 años de edad, con una media de edad de 41.6 años (desviación estándar: 11.25), de ellos el 60.9\% (n = 213) presentó edades entre 19-44 años. Predominó el sexo femenino, con 286 mujeres (81.7\%), sobre el masculino, con 64 hombres (18.3\%). De todos ellos, un $15.7 \%$ tenían sobrepeso y un $84.3 \%$ obesidad. El 98.3\% mostró obesidad abdominal y el $77.7 \%$ presentaba cifras de TA $\geq 130 / 80$ mmHg 35 o se encontraba bajo tratamiento antihipertensivo. Además, el 21.0 y el 28.3\% presentaron prediabetes e hipertrigliceridemia (HTG), respectivamente (Tabla 1).

EI FHTOA fue el más frecuente de los binomios estudiados en estos sujetos con sobrepeso y obesidad. La frecuencia del FHTOA fue del $76.9 \%$, seguido del FHGOA y el FHGLOA, con un 28.0 y un $20.9 \%$, respectivamente, mientras que la frecuencia de personas con SM fue del 69.1\% (Tabla 2). Se encontró que la proporción de individuos con el FHTOA y el FHGOA fue superior en el sexo masculino en relación con el femenino (90.6 vs. 73.8\%; $p=0.004 \mathrm{y}$ 42.2 vs. $24.8 \% ; p=0.005$, respectivamente). En cambio, el número de personas con SM y FHGLOA no difirió entre ambos sexos (Tabla 2).

La frecuencia de HTA e HTG fue superior en los hombres en comparación con las mujeres: HTA 90.6\% (58/64) vs. $74.8 \%$ (214/286), p $=0.0047$ e HTC $42.2 \%$ (27/64) vs. $25.2 \%$ (72/286), $p=0.0088$, respectivamente. 
TABLA 1. Características antropométricas, clínicas y bioquímicas de las personas estudiadas

\begin{tabular}{|c|c|}
\hline $\begin{array}{l}\text { Características clínicas, } \\
\text { antropométricas y bioquímicas }\end{array}$ & $\begin{array}{c}n(\%) \\
(n=350)\end{array}$ \\
\hline \multicolumn{2}{|l|}{ Edad (años) } \\
\hline $19-44$ & $213(60.9)$ \\
\hline$\geq 45$ & $137(39.1)$ \\
\hline \multicolumn{2}{|l|}{ Sexo } \\
\hline Femenino & $286(81.7)$ \\
\hline Masculino & $64(18.3)$ \\
\hline \multicolumn{2}{|l|}{ Color de la piel } \\
\hline Blanca & $212(60.6)$ \\
\hline Negra & $69(19.7)$ \\
\hline Mestiza & $69(19.7)$ \\
\hline \multicolumn{2}{|l|}{ IMC } \\
\hline Sobrepeso & $55(15.7)$ \\
\hline Obeso & $295(84.3)$ \\
\hline $\begin{array}{l}\text { Circunferencia de la cintura } \\
\text { alterada* }\end{array}$ & $344(98.3)$ \\
\hline $\begin{array}{l}\text { Tensión arterial }{ }^{\dagger}(\geq 130 / 80 \mathrm{mmHg} \\
\text { o tratamiento) }\end{array}$ & $272(77.7)$ \\
\hline Glucosa en ayunas alterada ${ }^{\ddagger}$ & $74(21.1)$ \\
\hline Triglicéridos elevados $§$ & $99(28.3)$ \\
\hline C-HDL bajo? & $232(66.3)$ \\
\hline
\end{tabular}

*Circunferencia de la cintura alterada: hombres $\geq 90 \mathrm{~cm}$; mujeres $\geq 80 \mathrm{~cm}^{3,33,34}$.

'Tensión arterial: $\geq 130 / 80 \mathrm{mmHg} 0$ tratamiento antihipertensión ${ }^{35}$.

FGlucosa en ayunas alterada: $\geq 5.6(100 \mathrm{mg} / \mathrm{dl})$ y $\leq 6.9 \mathrm{mmol} / \mathrm{l}(125 \mathrm{mg} / \mathrm{dl})$

o tratamiento para la hiperglucemia 3,33 .

§Triglicéridos elevados: $\geq 1.7 \mathrm{mmol} / \mathrm{l}(150 \mathrm{mg} / \mathrm{dl})$ o tratamiento para la dislipidemia 3,33

"C-HDL bajo: $<1.03 \mathrm{mmol} / \mathrm{l}$ en hombres ( $40 \mathrm{mg} / \mathrm{dl})$ y $<1.29 \mathrm{mmol} / \mathrm{l}$ ( $50 \mathrm{mg} / \mathrm{dl}$ ) en mujeres o tratamiento para la dislipidemia3,33.

C-HDL: colesterol de lipoproteínas de alta densidad; IMC: índice de masa corporal; n: número de casos con la condición.

Además, encontramos que la frecuencia de individuos con el FHGOA fue superior en las personas con color de piel blanca en relación con aquellas con color de piel negra y mestiza $(p=0.015)$ (Tabla 3$)$. Este resultado concuerda con la mayor frecuencia de HTG observada en personas con color de piel blanca en comparación con las personas de color de piel negra y mestiza: HTG 33.0\% (piel blanca,
TABLA 2. Distribución de individuos con sobrepeso y obesos según fenotipos y presencia de SM

\begin{tabular}{|l|c|c|c|}
\hline & $\begin{array}{c}\text { Total de } \\
\text { personas } \\
(\mathbf{n}=350)\end{array}$ & $\begin{array}{c}\text { Sexo } \\
\mathbf{f e m e n i n o} \\
(\mathbf{n}=\mathbf{2 8 6})\end{array}$ & $\begin{array}{c}\text { Sexo } \\
\text { masculino } \\
(\mathbf{n}=64)\end{array}$ \\
\hline FHTOA n (\%) & $269(76.9)$ & $211(73.8)$ & $58(90.6)^{*}$ \\
\hline FHGOA n (\%) & $98(28.0)$ & $71(24.8)$ & $27(42.2)^{\dagger}$ \\
\hline FHGLOA n (\%) & $73(20.9)$ & $54(18.9)$ & $19(29.7)$ \\
\hline SM según JIS n (\%) & $242(69.1)$ & $196(68.5)$ & $46(71.9)$ \\
\hline
\end{tabular}

* $p=0.004$ vs. sexo femenino, Chi cuadrada de Pearson.

${ }^{\dagger} p=0.005$ vs. sexo femenino, Chi cuadrada de Pearson.

n: número de casos con la condición; FHTOA: fenotipo hipertensiónobesidad abdominal; FHGOA: fenotipo hipertrigliceridemia-obesidad abdominal; FHGLOA: fenotipo hiperglucemia-obesidad abdominal; SM: síndrome metabólico; JIS: Joint Interim Statement ${ }^{3,33}$.

TABLA 3. Distribución de individuos con exceso de peso según fenotipos y presencia de SM de acuerdo con el color de la piel

\begin{tabular}{|c|c|c|c|c|}
\hline & \multicolumn{3}{|c|}{$\begin{array}{c}\text { Color de la piel } \\
(\mathbf{n}=350)\end{array}$} & \multirow{2}{*}{$\begin{array}{c}\text { Valor } \\
\text { de } \mathbf{p}^{*}\end{array}$} \\
\cline { 2 - 4 } & $\begin{array}{c}\text { Blanca } \\
\mathbf{n = 2 1 2}\end{array}$ & $\begin{array}{c}\text { Negra } \\
\mathbf{n}=69\end{array}$ & $\begin{array}{c}\text { Mestiza } \\
\mathbf{n}=69\end{array}$ & \\
\hline $\begin{array}{c}\text { FHTOA } \\
\mathrm{n}(\%)\end{array}$ & $154(72.6)$ & $56(81.2)$ & $59(85.5)$ & 0.057 \\
\hline $\begin{array}{c}\text { FHGOA } \\
\mathrm{n}(\%)\end{array}$ & $69(32.5)$ & $10(14.5)$ & $19(27.5)$ & 0.015 \\
\hline $\begin{array}{c}\text { FHGLOA } \\
\mathrm{n}(\%)\end{array}$ & $45(21.2)$ & $10(14.5)$ & $18(26.1)$ & 0.240 \\
\hline $\begin{array}{c}\text { SM según } \\
\text { JIS n (\%) }\end{array}$ & $147(69.3)$ & $45(65.2)$ & $50(72.5)$ & 0.651 \\
\hline
\end{tabular}

n: número de casos con la condición; FHTOA: fenotipo hipertensiónobesidad abdominal; FHGOA: fenotipo hipertrigliceridemia-obesidad abdominal; FHGLOA: fenotipo hiperglucemia-obesidad abdominal;

SM: síndrome metabólico; JIS: Joint Interim Statement3,33.

*Chi cuadrada de Pearson.

70/212), $14.5 \%$ (piel negra, 10/69) y 27.5\% (piel mestiza, 19/69); $p=0.012$, respectivamente.

Del mismo modo, la frecuencia del FHTOA tiene tendencia a ser superior en las personas con color de piel negra y mestiza, aun cuando no es significativamente diferente, en relación con aquellas con color de piel blanca (Tabla 3). Al analizar la presencia de 
HTA por color de la piel no observamos diferencias significativas (73.6\% [color de piel blanca, 156/212], $82.6 \%$ [color de piel negra, 57/69] y $85.5 \%$ [color de piel mestiza 59/69]; $p=0.065$, respectivamente).

En el grupo con FHTOA, la frecuencia de personas con SM fue mayor que en el grupo sin el FHTOA: 81.4 vs. $28.4 \%, p<0.001$ (Tabla 4). Igualmente, la proporción de personas con SM mostró ser superior en los sujetos con el FHGOA con relación con aquellos sin el FHGOA: 93.9 vs. 59.5\%, p < 0.0001. Al analizar los sujetos con el FHGLOA, la frecuencia de personas con el SM fue también elevada en comparación con aquellas sin el FHGLOA: 100 vs. 61.0\%, $\mathrm{p}<0.0001$ (Tabla 4).

Del total de sujetos diagnosticados con SM según los criterios de la JIS ( $n=242)$, el FHTOA detectó el mayor número de personas con SM $(n=219)$ en relación con el resto de los fenotipos (FHGOA $[\mathrm{n}=92]$ y FHGLOA [ $\mathrm{n}=73]$, respectivamente) (Tabla 4).

EI FHTOA identificó la presencia de sujetos con SM con una alta sensibilidad (90.5\%) y una especificidad del $53.7 \%$, así como un VPP del $81.4 \%$. La sensibilidad y VPN del FHGOA y el FHGLOA fueron inferiores a los del FHTOA, aunque ambos fenotipos (FHGOA y FHGLOA) mostraron una elevada especificidad y VPP (Tabla 5).
TABLA 4. Frecuencia de personas con SM de acuerdo con la presencia o no de los fenotipos estudiados

\begin{tabular}{|c|c|c|c|}
\hline \multirow[t]{2}{*}{ Fenotipos } & \multicolumn{2}{|c|}{$S M(n=350)$} & \multirow[t]{2}{*}{ Valor de $\mathrm{p}$} \\
\hline & $\begin{array}{l}\text { Con SM } \\
n=242\end{array}$ & $\begin{array}{l}\text { Sin SM } \\
\mathrm{n}=108\end{array}$ & \\
\hline FHTOA + $(n=269)$ & $219(81.4 \%)$ & $50(18.6 \%)$ & \multirow{2}{*}{$<0.001$} \\
\hline FHTOA - $(n=81)$ & $23(28.4 \%)$ & $58(71.6 \%)$ & \\
\hline $\mathrm{FHGOA}+(n=98)$ & $92(93.9 \%)$ & $6(6.5 \%)$ & \multirow{2}{*}{$<0.0001$} \\
\hline FHGOA $-(n=252)$ & $150(59.5 \%)$ & $102(40.5 \%)$ & \\
\hline FHGLOA + $(n=73)$ & $73(100 \%)$ & $0(0.0 \%)$ & \multirow{2}{*}{$<0.0001$} \\
\hline FHGLOA $-(n=277)$ & $169(61.0 \%)$ & $108(39.0 \%)$ & \\
\hline \multicolumn{4}{|c|}{$\begin{array}{l}\text { n: número de casos con la condición; FHTOA: fenotipo hipertensión- } \\
\text { obesidad abdominal; FHGOA: fenotipo hipertrigliceridemia-obesidad } \\
\text { abdominal; FHGLOA: fenotipo hiperglucemia-obesidad abdominal; } \\
\text { SM: síndrome metabólico. }\end{array}$} \\
\hline
\end{tabular}

El análisis del grado de concordancia diagnóstica entre las personas con SM según JIS y los tres fenotipos (FHTOA, FHGOA y FHGLOA) se muestra en la tabla 6. La concordancia entre los criterios de la JIS y el FHTOA fue moderada (0.475), así como aceptable para los FHGOA y FHGLOA (0.237 y 0.210, respectivamente).

\section{DISCUSIÓN}

Es importante enfatizar que la primera y única organización que consideró a la CC como criterio obligatorio fue la IDF. Según su definición de SM, una

TABLA 5. Precisión del diagnóstico de los distintos fenotipos para identificar personas con SM

\begin{tabular}{|l|c|c|c|c|}
\hline \multicolumn{5}{|c|}{ SM } \\
\hline Fenotipos de cintura & $\begin{array}{c}\text { Sensibilidad \% } \\
(\text { IC 95\%) }\end{array}$ & $\begin{array}{c}\text { Especificidad \% } \\
\text { (IC 95\%) }\end{array}$ & $\begin{array}{c}\text { VPP \% } \\
(\text { IC 95\%) }\end{array}$ & $\begin{array}{c}\text { VPN \% } \\
\text { (IC 95\%) }\end{array}$ \\
\hline FHTOA & $90.5(86.1-93.9)$ & $53.7(43.8-63.4)$ & $81.4(76.2-85.8)$ & $71.6(60.6-81.1)$ \\
\hline FHGOA & $38.0(31.9-44.5)$ & $94.4(88.3-98.0)$ & $93.9(87.1-97.7)$ & $40.5(34.3-46.9)$ \\
\hline FHGLOA & $30.2(24.5-36.4)$ & $100(96.6-100)$ & $100(95.1-100)$ & $39.0(33.2-45.0)$ \\
\hline
\end{tabular}

VPP: valor predictivo positivo; VPN: valor predictivo negativo; IC: intervalo de confianza; FHTOA: fenotipo hipertensión-obesidad abdominal; FHGOA: fenotipo hipertrigliceridemia-obesidad abdominal; FHGLOA: fenotipo hiperglucemia-obesidad abdominal; SM: síndrome metabólico. 
TABLA 6. Análisis de concordancia diagnóstica por coeficiente kappa entre los distintos fenotipos y SM

\begin{tabular}{|l|l|c|c|l|}
\hline \multicolumn{2}{|c|}{} & Kappa* $^{*}$ & $\begin{array}{c}\text { Error } \\
\text { estándar }\end{array}$ & $\begin{array}{l}\text { Grado de } \\
\text { concordancia }\end{array}$ \\
\hline \multirow{3}{*}{ SM } & FHTOA & 0.475 & 0.052 & Moderada \\
\cline { 2 - 5 } & FHGOA & 0.237 & 0.033 & Aceptable \\
\cline { 2 - 5 } & FHGLOA & 0.210 & 0.027 & Aceptable \\
\hline
\end{tabular}

*Concordancia: pobre $(\leq 0.00)$, leve (0.01-0.20), aceptable (0.21-0.40), moderada (0.41-0.60), considerable (0.61-0.80) y casi perfecta (0.81-1.00) ${ }^{36}$. FHTOA: fenotipo hipertensión-obesidad abdominal; FHGOA: fenotipo hipertrigliceridemia-obesidad abdominal; FHGLOA: fenotipo hiperglucemiaobesidad abdominal; SM: síndrome metabólico.

persona debe tener una obesidad abdominal como condición necesaria (CC alterada) y dos o más de los siguientes factores de riesgo: niveles altos de triglicéridos, C-HDL bajo, HTA y disglucemia37.

Debido a que el SM se presenta con amplias variaciones fenotípicas se han realizado estudios que asocian la obesidad abdominal con el resto de los componentes del SM (hipertensión, dislipidemia y disglucemia) ${ }^{11-15,31,34,38-41}$.

Los resultados de nuestro estudio muestran que el FHTOA fue el más frecuente de los binomios estudiados en sujetos con sobrepeso y obesidad, coincidiendo con Nita, et al., que han demostrado la utilidad de la combinación del binomio hipertensión-obesidad abdominal para detectar individuos con SM ${ }^{21}$.

La frecuencia del FHTOA en adultos con exceso de peso $(76.9 \%)$ es superior a la encontrada por Nita, et al. en población general $(43.3 \%)^{21}$, lo cual es racional, debido a que la HTA está considerablemente asociada con la obesidad abdominal (adiposidad central)13,14,38-40.

La alta proporción de hombres con el FHTOA y el FHGOA en este estudio se debe a que la frecuencia tanto de la HTA como de la HTG fue superior en los hombres en comparación con las mujeres. Datos similares con relación a la alta frecuencia de HTA y de HTG en hombres se han encontrado en algunos estudios en Cuba23,27,28,31 y Latinoamérica ${ }^{29,30}$.

Los datos de este estudio muestran también que la frecuencia del FHGOA fue superior en las personas de color de piel blanca que en la negra y mestiza. En Cuba existen controversias sobre la relación del FHGOA con el color de piel blanca ${ }^{17,42}$. Por el contrario, en el resto de los fenotipos y el SM ${ }^{27}$ no existió asociación con el color de la piel. Orduñes, et al.43 no encontraron diferencias en la frecuencia de hipertensión por el color de la piel en Cuba.

Respecto al análisis de la presencia de personas con SM en relación con los FHGOA y FHGLOA, casi todos son diagnosticados como SM, en cambio los sujetos sin dichos fenotipos también presentaban un suficiente número de personas con SM (59.5 y 61.0\%, respectivamente). En cambio, esto no ocurre con el FHTOA, donde la mayoría de estos sujetos concuerdan con el diagnóstico de personas con SM (81.4\%), mientras que en los individuos sin el FHTOA la proporción de personas con SM es mucho más baja (28.4\%).

Este estudio mostró, además, la alta sensibilidad (90.5\%) del FHTOA en la detección de personas con SM, así como VPP y VPN altos para el mismo propósito. Los valores altos de sensibilidad y de VPN para el FHTOA, en comparación con los calculados para los FHGOA y FHGLOA, orientan a que la asociación del binomio hipertensión-obesidad abdominal se utilice como herramienta para la detección de personas con SM. Aunque el estudio de Nita, et al. ${ }^{21}$ se hizo en población general, el FHTOA presentó también una alta sensibilidad (80.4\%) y VPP (84\%) para identificar personas con SM. Además, el FHTOA fue el que mostró una concordancia diagnóstica superior para identificar personas con SM en relación 
con los otros dos fenotipos analizados (FHGOA y FHGLOA).

Notamos que la presencia del FHTOA mostró una cantidad considerable de falsos positivos (especificidad del $53.7 \%$ ) y reveló una alta probabilidad para descartar realmente los sujetos que no tengan SM (VPN del 71.6\%). Al contrario, los FHGOA y FHGLOA exhibieron baja sensibilidad y bajo VPN, por tanto, estos fenotipos no descartan realmente a las personas con SM. No obstante, estos dos últimos fenotipos detectaron menos falsos positivos (especificidad de 84.4 y $100 \%$, respectivamente) y ostentaron VPP bien altos, por tal razón tienen una probabilidad superior a la del FHTOA para detectar realmente sujetos con diagnóstico de SM.

Nuestros resultados confirman que el FHTOA (alta sensibilidad) es útil para identificar o descartar a las personas con SM. Pues el no presentar el FHTOA descarta que probablemente la persona tenga SM. Esto quiere decir que la identificación de sujetos con el FHTOA da muy pocos falsos negativos, pero un número elevado de falsos positivos. Este último razonamiento es lógico, ya que podemos encontrar personas que presentan al momento del estudio solo el binomio hipertensión-obesidad abdominal y aún no ha aparecido el tercer componente del SM (confiriendo una especificidad baja). Por lo que urge tratar intensivamente la hipertensión y la obesidad ${ }^{9}$ en las personas con el FHTOA para evitar la evolución hacia el SM.

Es importante destacar que de las 16 posibles combinaciones (tres componentes del SM) para diagnosticar personas con SM según los criterios de la JIS, el binomio hipertensión-obesidad abdominal detecta la mayoría de los sujetos con SM (90.5\%; 219/242), binomio que se encuentra en siete de esas combinaciones, en cambio en las otras nueve combinaciones posibles de la JIS, que no incluyen a la
HTA, se detectan los otros 23 individuos con SM. Por consiguiente, el binomio hipertensión-obesidad abdominal es apropiado para identificar personas con SM.

En consecuencia, la combinación de ambos componentes (HTA y obesidad abdominal) es capaz de identificar a la mayoría de las personas con SM mucho mejor de lo que puede hacer el resto de los fenotipos estudiados (FHGOA y FHGLOA). La identificación del FHTOA en una persona puede ser una señal de alarma para el médico de los trastornos de los perfiles de lípidos y glucosa, así como del riesgo cardiovascular en el individuo $0^{12,15,38,41,44}$.

Las personas con los FHTOA y FHGOA mostraron una frecuencia superior de glucosa alterada en ayunas (GAA) en comparación con aquellos sin los fenotipos ( $p=0.030$ y $p=0.041$, respectivamente). Es decir, que del total de personas con GAA $(n=74)$, el FHTOA mostró mayor proporción de personas con esa condición (86.5\% [64/74]); en contraste, el FHGOA solo detecta al 37.8\% (28/74) de las personas con GAA. Estos resultados revelan al FHTOA como potencial advertencia de prediabetes (GAA). Otros investigadores también han encontrado resultados similares a la relación del FHGOA con la presencia de disglucemias ${ }^{16,19,20}$. Por otra parte, en la literatura revisada no encontramos información sobre la asociación del FHTOA y prediabetes.

Alrededor del SM hay controversia en cuanto a los mecanismos que conectan los factores de riesgo metabólico y la resistencia a la insulina, además de la discusión respecto a si es una entidad independiente y cuáles deberían ser los criterios para su diagnóstico ${ }^{1,8,9,31,45}$. Por ende, se sugiere que una forma práctica y sencilla para detectar sujetos con SM es comenzar con parámetros clínicos ordinarios fáciles de determinar, como la hipertensión y la obesidad abdominal ${ }^{21}$. 
En la práctica clínica diaria las determinaciones de glucemia y triglicéridos se podrán realizar cuando se encuentren sujetos con el FHTOA ${ }^{21}$. La identificación de un valor alterado de triglicéridos y/o de glucemia en sujetos con el FHTOA detectará a personas con otros componentes del $\mathrm{SM}^{21}$. Por tanto, se recomienda a las personas con el FHTOA que se comprueben regularmente los perfiles de lípidos y de glucosa.

En particular, la medición del FHTOA es una herramienta fácil de aplicar y no requiere de exámenes de laboratorio para el diagnóstico oportuno de personas con SM. Esto trae consigo implicaciones económicas en el sistema de salud, pues se evitaría la necesidad de pesquisa de perfil lipídico y de determinaciones de glucemia en la etapa inicial en personas con sobrepeso y obesidad.

La limitación principal de este estudio radica en que, al haber estudiado sujetos con exceso de peso que acudieron de manera consecutiva a un proyecto de investigación, el muestreo no es probabilístico y la muestra de pacientes no es representativa para que los resultados sean extrapolados a la población general. No obstante, la importancia del estudio radica en que, hasta donde conocemos, no existen estudios en Cuba en los cuales se compare la sensibilidad, especificidad y concordancia diagnóstica entre los distintos fenotipos estudiados (FHTOA, FHGOA, FHGLOA) para identificar personas con SM según los criterios JIS en población general, ni en población con exceso de peso. Sin embargo, Nita, et al. ${ }^{21}$, al estudiar una población general rumana con una muestra de 1,294 personas encontró una frecuencia del FHTOA mucho más baja (43.3\%) que nuestro estudio, pero con una alta sensibilidad de este fenotipo para identificar individuos con SM cercana a la nuestra.
En conclusión, su alta sensibilidad y alto valor predictivo negativo, así como la moderada concordancia del FHTOA para identificar personas con SM, y la sencillez en su determinación (consistente en la medición de la presión arterial y la circunferencia de cintura) lo hacen un buen candidato para ser utilizado como una opción para identificar o descartar personas con SM. La aplicación de este binomio es económica, pues no utiliza mediciones de laboratorio. EI FHTOA pudiera ser una alternativa para detectar personas con SM en poblaciones de riesgo para DM2 y ECV. Por lo tanto, recomendamos realizar otros estudios semejantes en Cuba con mayor número de participantes que sustenten estos resultados, de modo que puedan ser generalizables.

\section{AGRADECIMIENTOS}

Los autores agradecen a los especialistas del laboratorio de bioquímica del Instituto de Endocrinología por su excelente colaboración.

Este trabajo constituye parte de un proyecto de tesis de terminación de la Residencia en Endocrinología. Nuestro agradecimiento a los participantes en el estudio por su valiosa disposición.

\section{FINANCIAMIENTO}

Este estudio fue financiado por el Instituto de Endocrinología, La Habana, Cuba.

\section{CONFLICTO DE INTERESES}

Los autores declaran no tener conflictos de intereses. 


\section{RESPONSABILIDADES ÉTICAS}

\author{
Protección de personas y animales. Los autores de- \\ claran que para esta investigación no se han realiza- \\ do experimentos en seres humanos ni en animales.
}

\section{Confidencialidad de los datos. Los autores declaran que en este artículo no aparecen datos de pacientes.}

\section{Derecho a la privacidad y consentimiento informa-}

do. Los autores han obtenido el consentimiento informado de los pacientes y/o sujetos referidos en el artículo. Este documento obra en poder del autor de correspondencia.

\section{BIBLIOGRAFÍA}

1. Saklayen MG. The global epidemic of the metabolic syndrome. Curr Hypertens Rep. 2018;20(2):12. [citado: 2 de Agosto 2018]. Disponible en: https://doi.org/10.1007/s11906-018-0812-z.

2. Wilson PW, D’Agostino RB, Parise H, Sullivan L, Meigs JB. Metabolic syndrome as a precursor of cardiovascular disease and type 2 diabetes mellitus. Circulation. 2005;112:3066-72.

3. Alberti KG, Eckel RH, Grundy SM, Zimmet PZ, Cleeman JI, Donato KA, et al. Harmonizing the metabolic syndrome: a joint interim statement of the International Diabetes Federation Task Force on Epidemiology and Prevention; National Heart, Lung, and Blood Institute; American Heart Association; World Heart Federation; International Atherosclerosis Society; and International Association for the Study of Obesity. Circulation. 2009;120:1640-5.

4. Cabrera-Rode E, Stusser B, Cálix W, Orlandi N, Rodríguez J, CubaDueñas I, et al. Concordancia diagnóstica entre siete definiciones de síndrome metabólico en adultos con sobrepeso y obesidad. Rev Peru Med Exp Salud Publica. 2017;34:19-27.

5. Ranasinghe $P$, Mathangasinghe $Y$, Jayawardena R, Hills AP, Misra A. Prevalence and trends of metabolic syndrome among adults in the Asian-pacific region: a systematic review. BMC Public Health. 2017; 17(1):101. [citado: 28 de marzo 2020]. Disponible en: https://bmcpublichealth.biomedcentral.com/track/pdf/10.1186/s12889-0196711-7

6. Fanning E, O'Shea D. Genetics and the metabolic syndrome. Clin Dermatol. 2018;36:9-13.

7. Kuneš J, Vaněčková I, Mikulášková B, Behuliak M, Maletínská I, Zicha J. Epigenetics and a new look on metabolic syndrome. Physiol Res. 2015;64:611-20.

8. Simmons RK, Alberti KG, Gale EA, Colagiuri S, Tuomilehto J, Qiao Q, et al. The metabolic syndrome: Useful concept or clinical tool? Report of a WHO Expert Consultation. Diabetologia. 2010;53:600-05.

9. Kahn R, Buse J, Ferrannini E, Stern M. The metabolic syndrome: time for a critical appraisal: joint statement from the American Diabetes Association and the European Association for the Study of Diabetes. Diabetes Care. 2005;28:2289-304.
10. Kwasny C, Manuwald U, Kugler J, Rothe U. Systematic review of the epidemiology and natural history of the metabolic vascular syndrome and its coincidence with type 2 diabetes mellitus and cardiovascular diseases in different European countries. Horm Metab Res. 2018;50: 201-08.

11. Kwon H, Kim D, Kim JS. Body fat distribution and the risk of incident metabolic syndrome: A longitudinal cohort study. Sci Rep. 2017; 7(1):10955 . [citado: 28 de marzo 2020]. Disponible en: https://www. ncbi.nlm.nih.gov/pmc/articles/PMC5591218/pdf/41598_2017_Article_9723.pdf

12. Schoettl T, Fischer IP, Ussar S. Heterogeneity of adipose tissue in development and metabolic function. J Exp Biol. 2018;221(Pt Suppl 1). pii: jeb162958 [citado: 28 de marzo 2020]. Disponible en: https://jeb. biologists.org/content/jexbio/221/Suppl_1/jeb162958.full.pdf.

13. De Oliveira CM, Ulbrich AZ, Neves FS, Dias FAL, Horimoto ARVR, Krieger JE, et al. Association between anthropometric indicators of adiposity and hypertension in a Brazilian population: Baepend Heart Study. PLoS One. 2017;12(10):e0185225. [citado: 28 de marzo 2020]. Disponible en: https://www.ncbi.nlm.nih.gov/pmc/articles/ PMC5638240/pdf/pone.0185225.pdf.

14. Ononamadu CJ, Ezekwesili CN, Onyeukwu OF, Umeoguaju UF, Ezeigwe OC, Ihegboro GO. Comparative analysis of anthropometric indices of obesity as correlates and potential predictors of risk for hypertension and prehypertension in a population in Nigeria. Cardiovasc J Afr. 2017;28:92-9.

15. Domínguez-Reyes T, Quiroz-Vargas I, Salgado-Bernabé AB, Salgado-Goytia L, Muñoz-Valle JF, Parra-Rojas I. Las medidas antropométricas como indicadores predictivos de riesgo metabólico en una población mexicana. Nutr Hosp. 2017;34:96-101.

16. Lemieux I, Pascot A, Couillard C, Lamarche B, Tchernof A, Alméras N, et al. Hypertriglyceridemic waist a marker of the atherogenic metabolic triad (hyperinsulinemia, hyperapolipoprotein B, small, dense LDL) in men? Circulation. 2000;102:179-84.

17. Morejón-Giraldoni A, Rivas-Alpízar E, Salas-García V, Benet-Rodríguez M. Prevalencia del fenotipo hipertrigliceridemia cintura abdominal alterada: resultados de la segunda medición de la iniciativa CARMEN. Revista Finlay [revista en Internet]. 2014;4(4): [citado: 28 de marzo 2020]. Disponible en: http://www.revfinlay.sld.cu/index.php/finlay/ article/view/321

18. Linares-Reyes E, Castillo-Minaya K, Ríos-Mino M, Huamán-Saavedra J. Estudio de correlación entre los diagnósticos de cintura hipertrigliceridémica y síndrome metabólico en adultos de Trujillo, Perú. Rev Peru Med Exp Salud Publica. 2014;31:254-60.

19. Nuñez-Cortes JM, Mantilla Morató T, Toro R, Millán Pérez J, Mangas Rojas A; Comité científico del registro de hipertrigliceridemias de la Sociedad Española de Arteriosclerosis. Síndrome metabólico en pacientes con fenotipo clínico de "cintura hipertrigliceridémica". Nutr Hosp. 2015;32:1145-52.

20. Ren $Y$, Liu $Y$, Sun $X$, Deng $K$, Wang $C$, Li L, et al. Hypertriglyceridemia-waist and risk of developing type 2 diabetes: The rural Chinese cohort study. Sci Rep. 2017;7(1):9072. [citado: 28 de marzo 2020]. Disponible en: https://www.nature.com/articles/s41598-017-09136-x. pdf.

21. Nita C, Hancu N, Rusu A, Bala C. Hypertensive waist: First step of the screening for metabolic syndrome. Metab Syndr Relat Disord. 2009; 7:105-10.

22. Benet $M$, Morejón AF, Espinosa AD, Landrove O, Peraza D, Ordúñez PO. Factores de riesgo para enfermedades crónicas en Cienfuegos, Cuba 2010. Resultados preliminares de CARMEN II. Medisur [Internet]. 2010;8(2) [citado: 26 de octubre 2018]. Disponible en: http:// www.medisur.sld.cu/index.php/medisur/arti cle/view/1166/70

23. Delgado HM, Lastre K, Valdés ML, Benet M, Morejón AF, Zerquera JR. Prevalencia de hipertensión arterial en el área I del municipio Cien- 
fuegos. Segunda medición de la iniciativa CARMEN: cifras alarmantes. Revista Finlay. 2015;5:4-11.

24. Bonet M, Varona P, Chang M, García RG, Suarez R, Arcía N, et al. III Encuesta Nacional de factores de riesgo y actividades preventivas de enfermedades no transmisibles. Cuba 2010-2011. La Habana: Editorial de Ciencias Médicas; 2015.

25. Ministerio de Salud Pública. Dirección nacional de estadísticas y registros médicos. Anuario estadístico de salud 2009 [Internet]. La Habana: Ministerio de Salud Pública; 2010. Disponible en: http://files. sld.cu/admonensalud/files/2010/07/anuario-estadistico-de-salud-2009. pdf

26. Ministerio de Salud Pública. Dirección nacional de estadísticas y registros médicos. Anuario estadístico de salud 2017 [Internet]. La Habana: Ministerio de Salud Pública; 2018. Disponible en: http://files.sld.cu/ dne/files/2018/04/Anuario-Electronico-Espa\%C3\%B10l-2017-ed-2018.pdf

27. Morejón A, Benet M, Díez E, García D, Salas V, Ordúñez P. Síndrome metabólico en un área de salud de Cienfuegos. Segunda medición de CARMEN. Revista Finlay [Internet]. 2011;1(1) [citado: 22 octubre 2019]. Disponible en: http://www.revfinlay.sld.cu/index.php/finlay/article/ view/24

28. Miguel-Soca PE, Rivas-Estévez M, Sarmiento-Teruel Y, Mariño-Soler AL, Marrero-Hidalgo M, Mosqueda-Batista L, et al. Prevalence of metabolic syndrome risk factors in adults in Holguín, Cuba (2004-2013). MEDICC Review. 2016;18(1-2). [citado: 28 de marzo 2020]. Disponible en: https://www.scielosp.org/pdf/medicc/2016.v18n1-2/28-33/en

29. Escobedo J, Schargrodsky H, Champagne B, Silva H, Boissonnet CP, Vinueza $\mathrm{R}$, et al. Prevalence of the metabolic syndrome in Latin America and its association with sub-clinical carotid atherosclerosis: the CARMELA cross sectional study. Cardiovasc Diabetol. 2009;8:52. [citado: 22 de octubre 2019]. Disponible en: https://www.ncbi.nlm.nih. gov/pmc/articles/PMC2760519/pdf/1475-2840-8-52.pdf

30. Wong-McClure RA, Gregg EW, Barceló A, Lee K, Abarca-Gómez L, Sanabria-López $L$, et al. Prevalence of metabolic syndrome in Central America: a cross-sectional population-based study. Rev Panam Salud Pública. 2015;38:202-8.

31. Kassi E, Pervanidou P, Kaltsas G, Chrousos G. Metabolic syndrome: definitions and controversies. BMC Med. 2011;9:48. [citado: 2 de agosto 2019] Disponible en: https://www.ncbi.nlm.nih.gov/pmc/articles/ PMC3115896/pdf/1741-7015-9-48.pdf

32. World Health Organization. Consultation on Obesity. Obesity: preventing and managing the global epidemic. Ginebra: World Health Organization, WHO Technical Report Series 894; 2000.

33. Samson SL, Garber AJ. Metabolic syndrome. Endocrinol Metab Clin North Am. 2014;43:1-23.

34. Díaz O, Hernández J, Domínguez E, Martínez I, Bosch Y, del Busto A, et al. Valor de corte de la circunferencia de la cintura como predictor de disglucemia. Rev Cubana Endocrinol. 2017; 28:1-15.
35. Whelton PK, Carey RM, Aronow WS, Casey Jr DE, Collins KJ, Dennison Himmelfarb C, et al. 2017 ACC/AHA/AAPA/ABC/ACPM/ACS/APhA/ASH/ ASPC/NMA/PCNA Guideline for the prevention, detection, evaluation, and management of high blood pressure in adults: A Report of the American College of Cardiology/American Heart Association Task Force on Clinical Practice Guidelines. J Am Coll Cardiol. 2018;71: e127-e248.

36. Landis JR, Koch GG. The measurement of observer agreement for categorical data. Biometrics. 1977;33(1):159-74.

37. Alberti KGMM, Zimmet PZ, Shaw J. Metabolic syndrome-a new world-wide definition. A Consensus Statement from the International Diabetes Federation. Diabet Med. 2006;23:469-80.

38. Javier Sangrós F, Torrecilla J, Giraldéz-García C, Carrillo L, Mancera J, Mur T, et al. Asociación de obesidad general y abdominal con hipertensión, dislipidemia y presencia de prediabetes en el estudio PREDAPS. Rev Esp Cardiol. 2018;71:170-7.

39. Shen C, Zhou Z, Lai S, Tao X, Zhao D, Dong W, et al. Urban-rural-specific trend in prevalence of general and central obesity, and association with hypertension in Chinese adults, aged $18-65$ years. BMC Public Health. 2019;19:661. [citado: 30 de marzo 2020]. Disponible en: https://www.ncbi.nlm.nih.gov/pmc/articles/PMC6543650/ pdf/128892019 Article 7018.pdf

40. Lin YA, Chen YJ, Tsao YC, Yeh WC, Li WC, Tzeng IS, et al. Relationship between obesity indices and hypertension among middle-aged and elderly populations in Taiwan: a community-based, cross-sectional study. BMJ Open. 2019;9:e031660. [citado: 30 de marzo 2020]. Disponible en: https://bmjopen.bmj.com/content/bmjopen/9/10/e031660. full.pdf

41. Alwardat N, Di Renzo L, de Miranda RC, Alwardat S, Sinibaldi Salimei P, De Lorenzo A. Association between hypertension and metabolic disorders among elderly patients in North Jordan. Diabetes Metab Syndr. 2018;12(5):661-6.

42. Morejón-Giraldoni AF, Benet-Rodríguez M, Salas-Rodríguez V, RivasÁlpizar E, Vásquez-Mendoza EM, Navarrete-Borrero AA. Fenotipo hipertrigliceridemia cintura abdominal alterada y su asociación con los factores de riesgo cardiovasculares. Rev Haban Cienc Med. 2018;17(6):949-64.

43. Ordúñez P, Kaufman JS, Benet M, Morejon A, Silva LC, Shoham DA, et al. Blacks and Whites in the Cuba have equal prevalence of hypertension: confirmation from a new population survey. BMC Public Health. 2013;13:169. [citado: 30 de marzo 2020]. Disponible en: https:// www.ncbi.nlm.nih.gov/pmc/articles/PMC3635894/pdf/1471-2458-13169.pdf

44. Kjeldsen SE. Hypertension and cardiovascular risk: General aspects. Pharmacol Res. 2018;129:95-9.

45. Kahn R. Metabolic syndrome, is it a syndrome? Does it matter? Circulation. 2007;115:1806-10. 